\title{
Periodic structures with spherical alumina particles: Transmission and reflection spectra
}

\author{
A.A. Miskevich, V.A. Loiko \\ B.I. Stepanov Institute of Physics of NAS of Belarus, \\ 68, Nezalezhnastsi ave., Minsk, 220072, Belarus, \\ E-mail:miskevic@dragon.bas-net.by,loiko@dragon.bas-net.by
}

\begin{abstract}
Coherent transmission and reflection coefficients of individual monolayers of spherical alumina particles in the wavelength range from 0.3 to $1.0 \mu \mathrm{m}$ and multilayers consisting of a set of such monolayers are computed. Quasi-crystalline approximation of the theory for multiple scattering of waves and the transfer matrix method are used. Three types of coherent transmittance minima inherent to close-packed regular disperse systems are singled out and described. Spectra of a glass plate with one-sided and double-sided coating with alumina particles are analyzed. Systems for creating antireflection coatings, diffuse light scattering structures, selective and nonselective filters of transmitted and reflected light are considered.
\end{abstract}

Keywords: alumina particle, display, periodic structure, photonic crystal, transmittance and reflectance.

Manuscript received 12.12.12; revised version received 25.02.13; accepted for publication 19.03.13; published online 25.06.13.

\section{Introduction}

Alumina (aluminium oxide, $\mathrm{Al}_{2} \mathrm{O}_{3}$ ) is a promising material for numerous applications in optics, optoelectronics, electromechanics, display technologies, etc. We consider spectra of spatially ordered systems made of spherical alumina particles.

One of the approximations allowing to describe light propagation through the layer of the spatiallyordered particles is the quasi-crystalline approximation (QCA) [1] of the statistical theory for multiple scattering of waves. In this approximation, the transmittance and reflectance are determined by the sum of waves scattered by particles with accounting of their re-irradiation and correlation of spatial location. Correlation is taken into account by the radial distribution function (RDF) [2].

The method of the radial distribution function for non-ideal planar photonic crystal (PPC) modeling has been described previously in [3]. Its essence is as follows. At first, we select the center of any particle as the coordinate origin and evaluate the radii of coordination circles of the ideal lattice and the number of particle centers located in each circle. The dependence of the number of particle centers on their distance in lattice is a set of the infinitely narrow peaks at distances which are equal to the coordination circles radii. Accordingly, the radial distribution function of an ideal photonic crystal (PC) has nonzero values at these distances and equals to zero at the other ones. Actual PCs typically have non-ideal lattice with "blurred" coordination circles. It was shown [3] that the expression for calculating the radial distribution function $\mathrm{g}(u)$ of the real PPC (consisting of spherical monodisperse particles) can be written as:

$$
\mathrm{g}(u)=\rho_{0}^{-1} \sum_{i} \frac{N_{i}}{2 \pi R_{i}} \frac{1}{\sqrt{2 \pi} \sigma(u)} \exp \left(-\frac{\left(u-R_{i}\right)^{2}}{2 \sigma^{2}(u)}\right) .
$$

Here, $\sigma(u)$ is the blurring function which characterizes the "blurring" (broadening) of coordination circles (into the fuzzy ring-shaped areas) as a function of the distance $u$ relatively to the coordinate origin. It is reasonable to use the linear blurring function: 
$\sigma(u)=\sigma_{0}(a u+b)$.

The function (2) takes into account asymmetry of individual peaks of RDF, which are observed in experiment. It allows to simulate RDFs of PCs in a wide range of their ordering degree. The RDFs obtained with using (2) are well adapted to be used in QCA. Moreover, the calculation of RDF and, consequently, coherent transmission and reflection coefficients of PPC in QCA, is fast and requires low amounts of computational resources. In the equations (1) and (2) $u=R / D$ is the dimensionless distance expressed in diameters $D$ of particles $(u \geq 1) ; R$ is the distance in a monolayer plane relatively to the coordinate origin; $\rho_{0}-$ averaged numerical particle concentration in the monolayer; $N_{i}-$ number of particle centers in the coordination circle with the radius $R_{i}$ of an ideal crystal; $\sigma_{0}-$ dimensionless initial dispersion of coordination circle radii expressed in particle diameters; $a$ and $b$ are the coefficients chosen as dependent on the filling coefficient (the ratio of the area of particle projections on the layer plane to the area where they are distributed) of layer, type of lattice, and $\sigma_{0}$. The ordering degree and scale of the spatial order of the simulated crystal can be specified by changing of $\sigma_{0}$, and $a$ and $b$ coefficients, respectively.

Note that at $\sigma(u)=$ const, Eq. (1) is transformed into the known expression for RDF which describes the Gaussian blurring of coordination circles in crystal lattice [4].

This work is the theoretical study of coherent transmittance and reflectance inherent to a layered system of plane-parallel monolayers (multilayer) of spatially-ordered monodisperse spherical alumina particles. The system is illuminated normally to the planes of monolayers. At first, in QCA $[1,3,5,6]$ the spectra of coherent transmission and reflection coefficients of individual monolayers are calculated. Then, using the obtained coefficients and the transfer matrix method (TMM) $[7,8]$ the coherent transmission and reflection coefficients of a multilayer are evaluated.

Spectra of coherent transmission and reflection coefficients of the glass plate with one- and double-sided coating with alumina particles are investigated. Antireflection coatings, selective and nonselective reflectors, transmission and reflection filters, diffuse scattering plates are considered as examples for possible application of the obtained results.

\section{Spectra of coherent transmission and refraction of monolayer}

Consider a plane-parallel monolayer of aluminium oxide particles. Spectral dependence of the real part of the refractive index for alumina is taken from the ref. [9]. Pay attention that imaginary part of the refractive index in the considered spectral range is zero.

The spectra of coherent transmission $T_{c}$ and reflection $R_{c}$ coefficients of regularly-packed monolayer (PPC) with hexagonal lattice and partially-ordered monolayer consisting of monodisperse spherical $\mathrm{Al}_{2} \mathrm{O}_{3}$ particles are shown in Fig. 1. Note that in this work, the notation "regularly-packed" designates non-ideal packing of particles in PPC's lattice. The monolayer filling coefficient $\eta=0.5$. Coherent transmission and reflection coefficients of monolayers were calculated in QCA $[1,3,5,6]$. The radial distribution function of PPC was evaluated by the method described schematically above with using the blurring function (2) for coordination circles as follows: $\sigma(u)=\sigma_{0} u, \sigma_{0}=0.01$. To simulate RDF of the partially-ordered monolayer of particles, the solution of Ornstein-Zernike integral equation [10] was used. It was numerically calculated in the Percus-Yevick approximation [11] for a system of hard spheres by the iteration method described in [12]. The obtained results demonstrate influence of spatial arrangement of particles on coherent transmittance and reflectance of monolayer. In the spectra of partiallyordered monolayers, the transmission minimum caused by interference of incident and scattered waves takes place. This minimum is situated in the vicinity of the extinction efficiency factor maximum. For a partiallyordered monolayer of monodisperse spherical $\mathrm{Al}_{2} \mathrm{O}_{3}$ particles with the diameter $D=0.3 \mu \mathrm{m}$ and the filling coefficient $\eta=0.5$, this minimum occurs at $\lambda \approx 0.357 \mu \mathrm{m}$ (see minimum 1' in the dashed line in Fig. 1a). Its depth and position depend on the concentration and optical properties of particles. Such a minimum also takes place in the monolayer with a regular packing of particles. However, its position is shifted relatively to the first one. For PPC with hexagonal lattice, it occurs at $\lambda \approx$ $0.339 \mu \mathrm{m}$ (see the minimum 1 in the solid line in Fig. 1a). Unlike the spectrum of the partially-ordered monolayer, in addition, the spectrum of PPC has a sharp deep minimum caused by the particle location periodicity. It can have a fine structure. For parameters indicated in the figure caption, this minimum is located in the wavelength range 0.52 to $0.53 \mu \mathrm{m}$ (see the minimum 2 in the solid line in Fig. 1a). There is also a sharp maximum of the coherent reflection coefficient (see the solid line in Fig. 1b) within the same range. Such sharp maxima observed in light diffraction by metallic gratings are known as Wood's anomalies [13]. Thus, at normal illumination of the planar monolayer of regularly-packed particles (planar photonic crystal), there is an effect similar to the photonic band gap effect, which takes place in PCs at light propagation along the periodicity of the multilayer structure.

The transmission minima $\left(1,1^{\prime}\right)$ can be very deep. Their values depend on the concentration and optical characteristics of particles. For example, the value of the transmission minimum 1 of PPC with hexagonal lattice of particles with the diameter $D=0.3 \mu \mathrm{m}$ is $T_{c} \approx 10^{-8}$ at the monolayer filling coefficient $\eta=0.533$ and wavelength $\lambda \approx 0.327 \mu \mathrm{m}$.

The particles are non-absorbing within the spectral range under consideration. As a result, the incoherent (diffuse) component $\Sigma_{\text {inc }}$ of light transmitted and 
reflected by a monolayer is equal to $\Sigma_{\text {inc }}=1-\left(T_{c}+R_{c}\right)$. The results of calculation of the coherent transmittance and reflectance sum $T_{c}+R_{c}$ for PPC and partially-ordered monolayer are displayed in Fig. 1b. For PPC at the wavelengths $\lambda \geq 0.52 \mu \mathrm{m}$, practically all the energy of incident light is either transmitted through the regularlypacked monolayer straight forward or reflected by it straight backward. Accordingly, the diffuse (incoherent) component is absent. Pay attention that the minimum 2 is located at the beginning of this spectral range.

Fraction of the incoherent component of radiation $\Sigma_{\text {inc }}$ transmitted and reflected by the partially-ordered monolayer is nonzero over the entire considered wavelength range. For the parameters indicated in the figure caption, the incoherent component $\Sigma_{i n c}$ is less than 0.1 at $\lambda \geq \sim 0.6 \mu \mathrm{m}$ and decreases with increasing the wavelength. With the filling coefficient decreasing, the value of $\Sigma_{i n c}$ decreases in this spectral range.
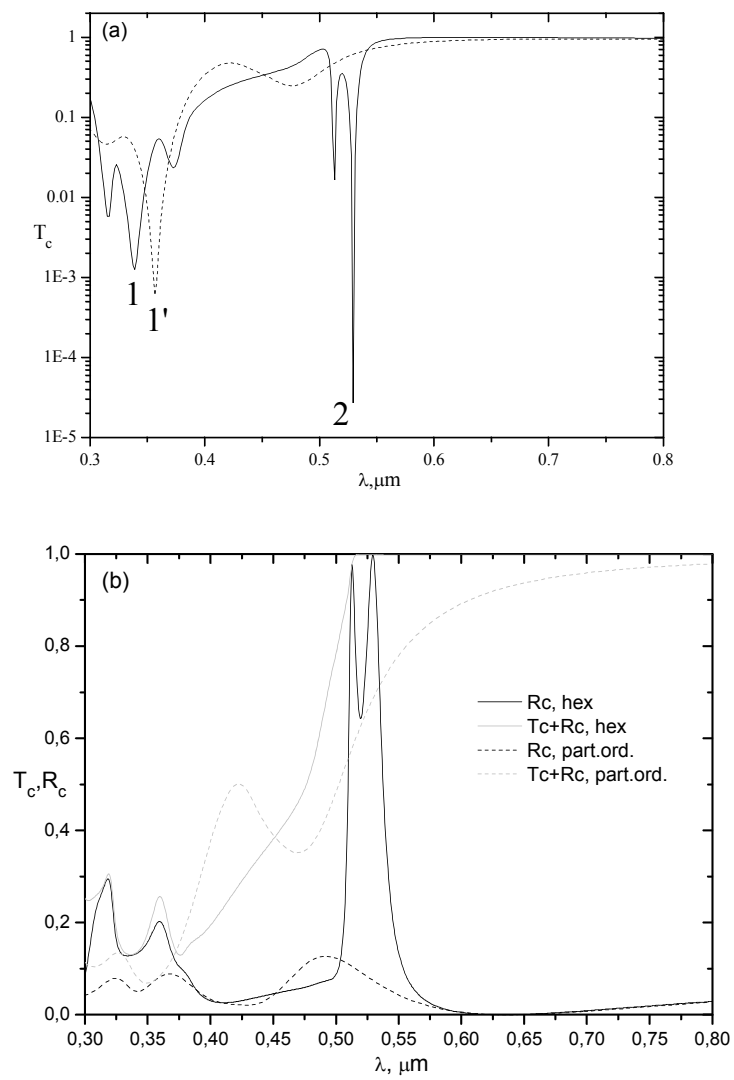

Fig. 1. Spectral dependences of the coherent transmission $T_{c}(a)$, reflection $R_{c}$ coefficients and their sum $T_{c}+R_{c}(b)$ for the monolayer of alumina particles: regularly-packed monolayer (PPC) with the hexagonal lattice (solid lines) and partiallyordered monolayer (dashed lines). Gray solid line is $T_{c}+R_{c}$ of regularly-packed monolayer, gray dashed line is $T_{c}+R_{c}$ of partially-ordered monolayer $(b)$. The monolayer filling coefficient $\eta=0.5$. The radial distribution function of PPC is calculated at $\sigma(u)=\sigma_{0} u, \sigma_{0}=0.01$. The particle diameter $D=$ $0.3 \mu \mathrm{m}$ for all the monolayers. The numbers $1,1^{\prime}$, and 2 indicate the types of the transmission minima (see description in the text).

\section{Light propagation in a multilayer}

Consider light propagation in a multilayer with taking into account multiple reflections between monolayers by using the transfer matrix method $[7,8]$. We suppose the monolayers as interfaces and the spaces between them as layers.

In Fig. 2, the coherent transmission and reflection coefficients of multilayer consisting of 12,30 and 60 monolayers with hexagonal packing of monodisperse spherical $\mathrm{Al}_{2} \mathrm{O}_{3}$ particles in air are displayed. The numerical calculation results were obtained for the spectral ranges where the value of incoherent component for the monolayer $\Sigma_{\text {inc }}=1-\left(T_{c}+R_{c}\right)$ was small (according to the results presented in Fig. $1 \mathrm{~b}$, the $\Sigma_{\text {inc }}$ value is small at $\lambda \geq 0.6 \mu \mathrm{m}$ ). The diameter of particles $D=0.3 \mu \mathrm{m}$, monolayers filling coefficient $\eta=0.5$, distances between adjacent monolayers $h=0.3 \mu \mathrm{m}$. The
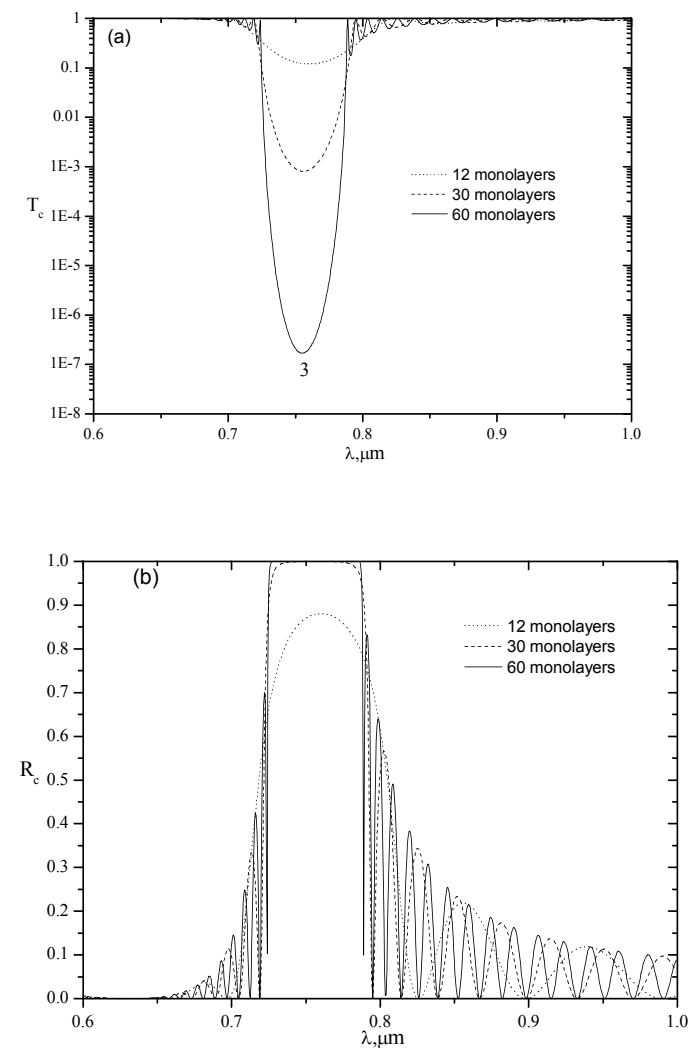

Fig. 2. Spectra of the coherent transmittance $T_{c}(a)$ and reflectance $R_{c}(b)$ of multilayer consisting of 12,30 , and 60 PPCs with hexagonal packing of $\mathrm{Al}_{2} \mathrm{O}_{3}$ particles in air. Distances between adjacent monolayers are $h=0.3 \mu \mathrm{m}$. The filling coefficient of each monolayer $\eta=0.5$, diameter of particles $D=0.3 \mu \mathrm{m}$. The radial distribution function of PPC is calculated at $\sigma(u)=\sigma_{0} u, \sigma_{0}=0.01$. The digit 3 indicates the transmission minimum (photonic band gap) inherent to the layered disperse system (see description in the text). 
calculation for the monolayer (in this case it is a planar photonic crystal) was implemented into QCA. The calculation for the multilayer was carried out using TMM. We name this combination of the methods as QCA-TMM.

The photonic band gap (PBG) caused by the multilayer periodic structure arises with increasing the number of monolayers. It is formed in the wavelength range $\sim 0.75$ to $0.77 \mu \mathrm{m}$ for the considered parameters of the system. See the transmission minimum indicated as 3 in Fig. 2a. As seen from the results in Fig. 2, already for 12 identical monolayers the noticeable PBG arises: $T_{c} \approx 0.12$ at $\lambda \approx 0.761 \mu \mathrm{m}$. For 30 monolayers, the coherent transmission coefficient $T_{c} \approx 8.1 \cdot 10^{-4}$ at $\lambda \approx$ $0.757 \mu \mathrm{m}$, and for 60 monolayers $T_{c} \approx 1.7 \cdot 10^{-7}$ at $\lambda \approx$ $0.756 \mu \mathrm{m}$. In the same spectral range, the coherent reflectance maximum comparable by its width with the coherent transmittance minimum 3 is formed.

As it follows from the results of calculations, these three types of coherent transmittance minima are inherent to the close-packed regular disperse system (see Fig. 1a where they are indicated by digits 1, 2, and digit 3 in Fig. 2a). The transmittance minimum 1 is caused by the concentration and optical properties of particles in a single monolayer. It takes place both for PPC and partially-ordered monolayer. The transmittance minimum 2 is caused by regularity in particle locations within a single monolayer. It takes place for PPC at sizes of particles and distances between them compared with the wavelength of the incident light. The transmittance minimum 3 is PBG. It takes place in spectra of the periodic system at wave propagation along periodicity of the multilayer structure.

The depths, widths, locations, and the number of PBGs of the multilayer depend on distances between its monolayers. Calculations for the same monolayer parameters as in Fig. 2 show: when the distance between monolayers is changed from 0.3 up to $0.5 \mu \mathrm{m}$, the depth and width of PBG are increased, and PBG location is shifted to the larger wavelengths. With further increasing the distance, location of this PBG is shifted to the larger wavelengths, and PBG depth decreases. Simultaneously, in the range of short wavelengths the additional more narrow PBGs appear. The longer is the distance between monolayers, the higher is the number of appearing PBGs. The results show that multilayers consisting of PPCs can be used to develop and manufacture the bandpass radiation filters in a wide spectral range.

Comparison with the results obtained for the partially-ordered monolayers has shown that the spectra of the system of partially-ordered monolayers and the system of PPCs have both general and different features.

In particular, for both systems (with the same particle diameter $D=0.3 \mu \mathrm{m}$ ) PBG formation within the range $\lambda \approx 0.73 \ldots 0.78 \mu \mathrm{m}$ and an increase in the number of spectral oscillations with the number of monolayers takes place. That is caused by periodicity of the multilayer structure along the direction of the incident wave and multiple wave reflections in a system. The absolute values of the minima of transmission in PBG differ slightly.

There are significant differences in PBG formation between the systems of planar photonic crystals and partially-ordered monolayers of particles. PBG of the PPCs system is more symmetric and has more sharp edges in comparison with PBG of partially-ordered monolayers system. The values of the reflection maxima in PBG are also noticeably different. Coherent reflectance maximum of the partially-ordered monolayers system in PBG $R_{c, \text { max }} \approx 0.81$ (at the wavelength $\lambda \approx 0.738 \mu \mathrm{m})$, whereas the respective maximum of the PPC system $R_{c, \max } \approx 1$.

\section{Glass plate covered with alumina particles: transmittance and reflectance spectrum}

The coefficient of light reflection by a plane-parallel glass plate in air is approximately $0.08(\sim 0.04$ from each glass-air interface). To reduce the reflection of light from the plate, the antireflection coatings on its surfaces are used. These are typically the thin dielectric layers of a certain refractive indices and thicknesses. It is promising to create such coatings from aluminum oxide $[14,15]$, due to their relative simplicity and low cost production.

Consider a two-layer system consisting of a homogeneous plane-parallel layer of aluminum oxide on a plane-parallel glass plate (Fig. 3).

Let us calculate the coherent transmission and reflection coefficients of this system under TMM. The technique for calculating the reflection and transmission of a system consisting of sufficiently thin layers is based on the use of complex amplitude reflection and transmission coefficients. When the system includes a thick layer (e.g., layer $L_{2}$ in Fig. 3), the energy transmission and reflection coefficients are used instead of the amplitude ones [7]. Coherent transmission and reflection coefficients of this system are shown in Fig. 4. In this figure, the transmission and reflection spectra of the plane-parallel glass plate without coating are shown as well.

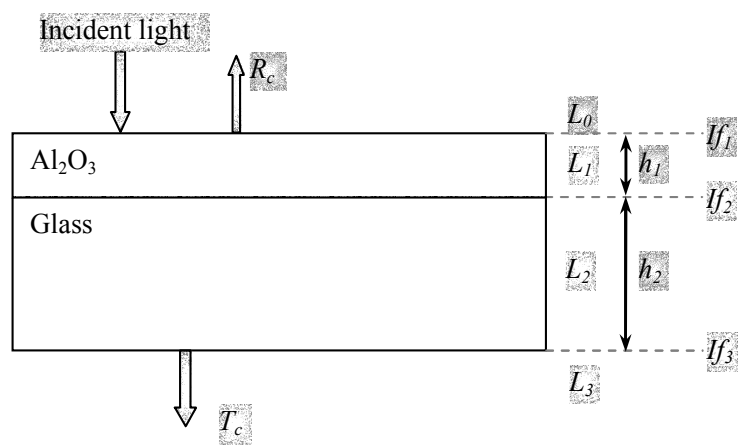

Fig. 3. Schematic presentation of homogeneous plane-parallel alumina layer on the surface of a plane-parallel glass plate (side view). 

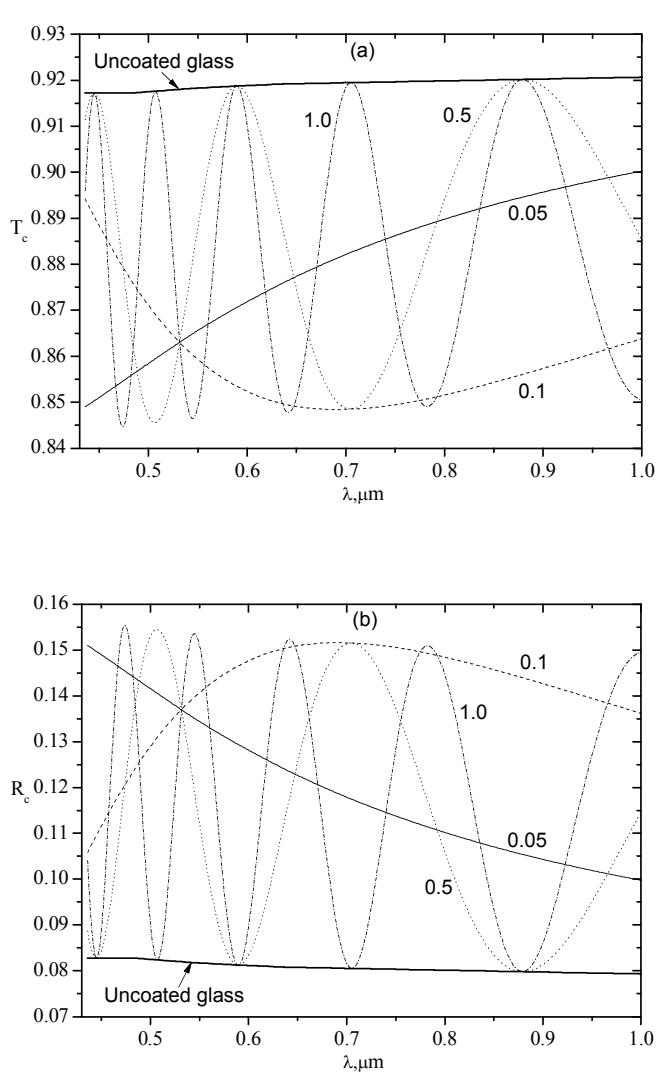

Fig. 4. Spectra of the coherent transmittance $T_{c}(a)$ and reflectance $R_{c}(b)$ of an uncoated plane-parallel glass plate (thick line) and system of plane-parallel homogeneous $\mathrm{Al}_{2} \mathrm{O}_{3}$ layer on the surface of glass plate (thin lines) at different thicknesses of $\mathrm{Al}_{2} \mathrm{O}_{3}$ layer. The thickness of glass plate is $2 \mathrm{~mm}$. Numbers near lines indicate the thicknesses of $\mathrm{Al}_{2} \mathrm{O}_{3}$ layer in micrometers.

The results displayed in Fig. 4 were obtained under the assumption that there are three interfaces $I f_{1} \ldots I f_{3}$ in the system (see Fig. 3). It was assumed that the interfaces are flat boundaries of $\mathrm{Al}_{2} \mathrm{O}_{3}$ layer $L_{1}$ (with the thickness $h_{1}$ ) and glass layer $L_{2}$ (with the thickness $h_{2}$ ). The amplitude reflection and transmission coefficients of planar interfaces between the layers are calculated by the Fresnel formulae [16]. The layer $L_{2}$ of glass has the thickness $h_{2}=2 \mathrm{~mm}$, which is much higher than the wavelengths under consideration. Therefore, $L_{2}$ is a "thick" layer, and the energy transmission and reflection coefficients are used accordingly to [7]. The layers $L_{0}$ and $L_{3}$ are semi-infinite layers of the environment (air). The refractive indexes of the Corning 1737 glass were used in the calculations [17]. As it follows from the results shown in the figure, coating of glass with the homogeneous layer of alumina does not reduce the coherent reflection coefficient of a system in comparison with that of the uncoated glass plate. Changes in the $\mathrm{Al}_{2} \mathrm{O}_{3}$ layer thickness lead to a change in the oscillation frequency of the transmission and reflection as a function of wavelength.

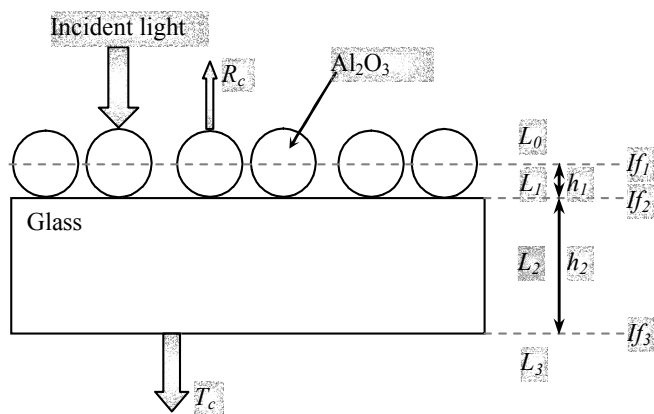

Fig. 5. Schematic presentation of system consisting of monolayer of monodisperse spherical $\mathrm{Al}_{2} \mathrm{O}_{3}$ particles on glass (side view).

Now consider a system consisting of a glass plate covered with a monolayer of $\mathrm{Al}_{2} \mathrm{O}_{3}$ particles on its upper surface (Fig. 5).

Let us calculate coherent transmission and reflection coefficients of this system using the scheme described above. Find the conditions under which a coating of alumina particles will serve as an antireflection layer (at normal illumination). Calculation of the coherent transmission and reflection coefficients of the system is fulfilled with the assumption that it consists of three interfaces: a monolayer of alumina particles $I f_{1}$ and the two flat interfaces of glass plate $I f_{2}$ and $I f_{3}$ (see Fig. 5). Accordingly, there are two layers between interfaces: $L_{1}$ with the thickness $h_{1}$ of air and $L_{2}$ with the thickness $h_{2}$ of glass. The thickness $h_{1}$ is equal to the radius of particles. Layers $L_{0}$ and $L_{3}$ are semiinfinite layers of the environment (air). Calculation of coherent transmission and reflection of interface $I f_{1}$ (planar PC) is carried out in QCA with $\sigma(u)=\sigma_{0} u$, $\sigma_{0}=0.01$, and of interfaces $I f_{2}$ and $I f_{3}$ (planar boundaries between the layers) by using the Fresnel formulae.

Depicted in Fig. 6 are the coherent transmission and reflection spectra of the uncoated glass plate and the plate coated with a monolayer possessing the hexagonal lattice of monodisperse spherical alumina particles. As it follows from the presented results, when the size of $\mathrm{Al}_{2} \mathrm{O}_{3}$ particles is much smaller than wavelengths, one can obtain an almost uniform spectrum with the reflectance smaller than the reflectance, and the transmittance larger than the transmittance of the uncoated glass plate. In the visible spectral range, when the coating monolayer contains $\mathrm{Al}_{2} \mathrm{O}_{3}$ particles with the diameter $D=0.1 \mu \mathrm{m}$, the reflection coefficient decreases down to $\sim 0.047$. With increasing the $\mathrm{Al}_{2} \mathrm{O}_{3}$ particle diameter up to $0.5 \mu \mathrm{m}$, the transmittance minimum and reflectance maximum in the spectrum within the range of wavelengths 0.85 to $0.88 \mu \mathrm{m}$ appear. They are caused by regularity in particle locations inside the monolayer. For sufficiently large particles, in some spectral ranges the values of the coherent reflectance are lower than the reflectance of the bare interface (for the glass-air boundary $R_{c} \approx 0.04$ ), and the coherent transmission coefficients are small. It implies that the major part of the radiation is incoherent. 

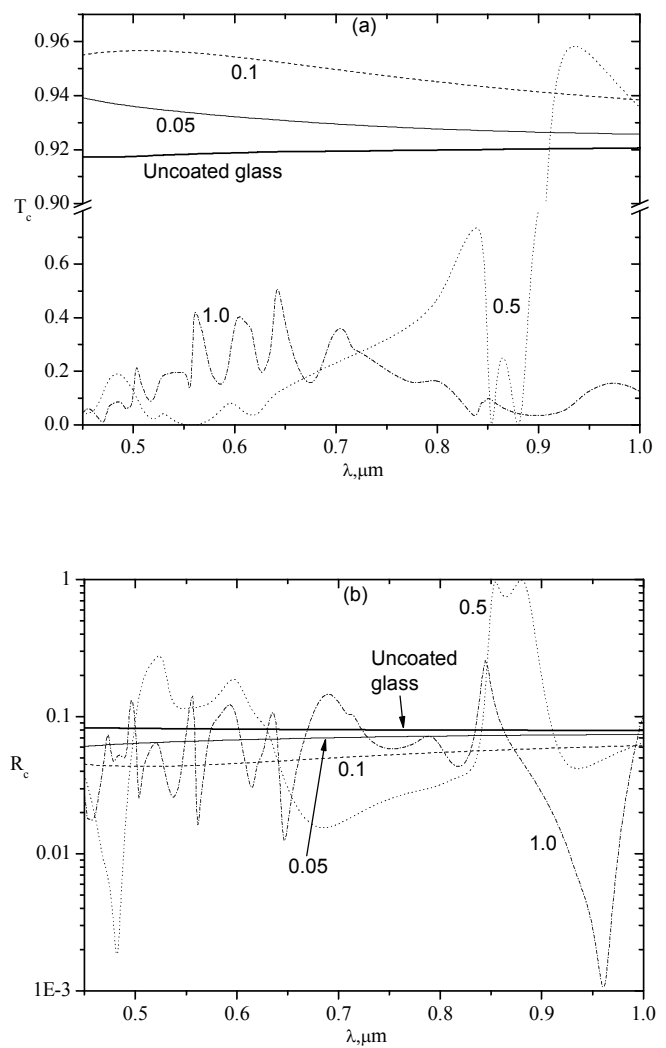

Fig. 6. Spectra of the coherent transmittance $T_{c}(a)$ and reflectance $R_{c}(b)$ of the uncoated plane-parallel glass plate (thick line) and system of monolayer with the hexagonal packing of monodisperse spherical $\mathrm{Al}_{2} \mathrm{O}_{3}$ particles on this plate for various particle sizes. The thickness of plate is $2 \mathrm{~mm}$, filling coefficient of monolayer $\eta=0.5$. Ciphers near the lines indicate diameters of $\mathrm{Al}_{2} \mathrm{O}_{3}$ particles in micrometers.

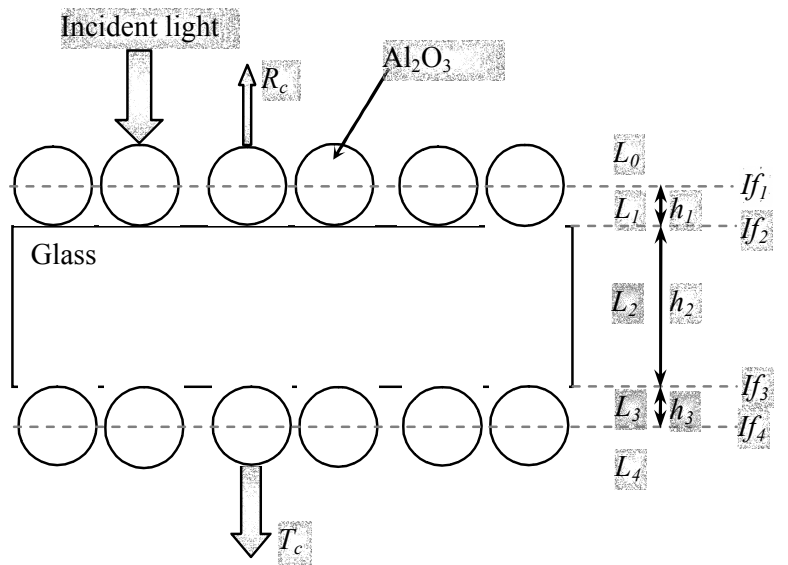

Fig. 7. Schematic presentation of multilayer system with two monolayers of monodisperse spherical $\mathrm{Al}_{2} \mathrm{O}_{3}$ particles on top and bottom surfaces of the glass plate.

To simultaneously reduce the coherent reflectance and increase the coherent transmittance, we should "exclude" reflections not only from the top, but also from the bottom interface of the plate. To solve this problem, consider the double-sided coating (Fig. 7). Using QCA-TMM, calculate the spectra of this system
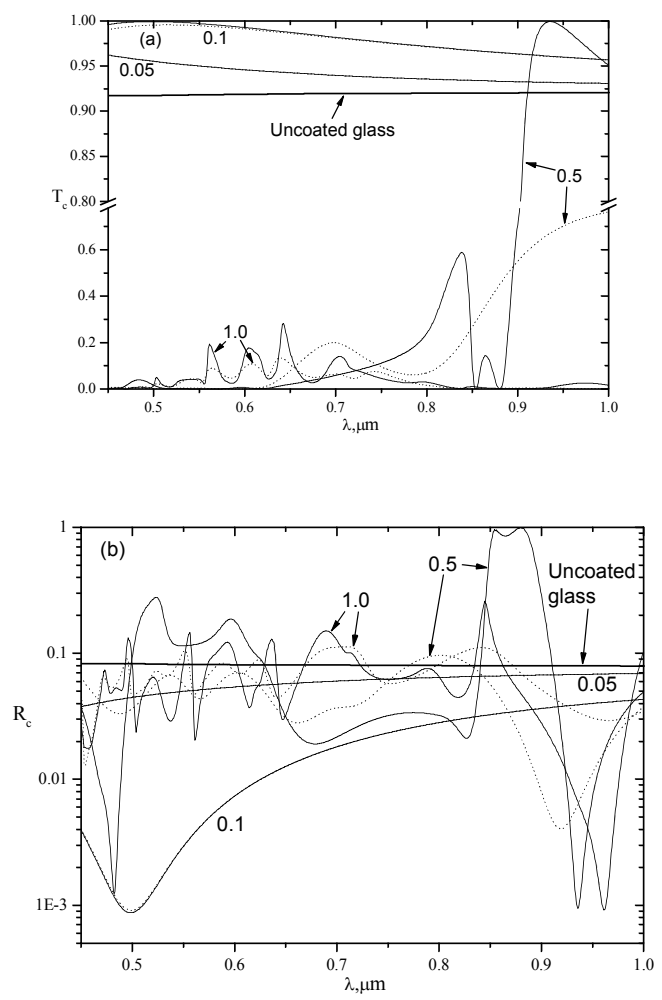

Fig. 8. Spectra of the coherent transmittance $T_{c}(a)$ and reflectance $R_{c}(b)$ of the uncoated plane-parallel glass plate (thick line) and system of identical partially-ordered (dotted lines) and regularly-packed monolayers with hexagonal packing of $\mathrm{Al}_{2} \mathrm{O}_{3}$ particles (thin solid lines, $\sigma(u)=\sigma_{0} u$, $\left.\sigma_{0}=0.01\right)$ on top and bottom surfaces of the glass plate for various particle sizes. The thickness of glass plate is $2 \mathrm{~mm}$, filling coefficient of monolayers $\eta=0.5$. Diameters (in micrometers) of particles are indicated near the lines.

when monolayers of aluminum oxide particles are regularly-packed and partially-ordered. Fig. 8 shows the spectral dependences of coherent transmittance and reflectance when the top and bottom monolayers of $\mathrm{Al}_{2} \mathrm{O}_{3}$ particles are identical. Thin solid lines depict the spectra of the system with the regularly-packed monolayers (PPCs with hexagonal lattice), the dashed lines show the spectra of systems with partially-ordered monolayers of $\mathrm{Al}_{2} \mathrm{O}_{3}$ particles. Pay attention that the displayed results have a good accuracy in the spectral range where the value of $T_{c}+R_{c} \approx 1$. As it follows from the calculation results, the coatings with monolayers of $\mathrm{Al}_{2} \mathrm{O}_{3}$ particles on both surfaces of the glass plate can significantly reduce the coherent reflection and increase the coherent transmission coefficients of the system as compared with those for the one-sided coated system. At a double-sided coating with identical monolayers of $\mathrm{Al}_{2} \mathrm{O}_{3}$ particles of diameter $D=0.1 \mu \mathrm{m}$, the calculated coherent reflection coefficient $R_{c}<0.025$ in the visible spectral range with the minimum lower than 0.01 . If the size of $\mathrm{Al}_{2} \mathrm{O}_{3}$ particles significantly smaller than the 
incident light wavelength, the differences in coherent transmittance and reflectance spectra of the systems coated with PPCs and partially-ordered monolayers are negligible (see lines for $D=0.05 \mu \mathrm{m}$ and $D=0.1 \mu \mathrm{m}$ in Fig. 8). If the particles size is comparable with the incident light wavelength, the differences in the spectra of systems with PPCs and partially-ordered monolayers become significant. They are more pronounced in the spectral range of the transmission minimum and reflection maximum (see solid lines for $D=0.5 \mu \mathrm{m}$ within the range of wavelengths $\sim 0.85 \mu \mathrm{m}<\lambda<\sim 0.88 \mu \mathrm{m}$ in Fig. 8) caused by the regularity of particle packing in a planar PC. It follows that if the creation of antireflection coatings from the $\mathrm{Al}_{2} \mathrm{O}_{3}$ particles does not required their high ordering, then for the construction of selective reflectors and transmission filters the ordering of particles in monolayers plays a crucial role.
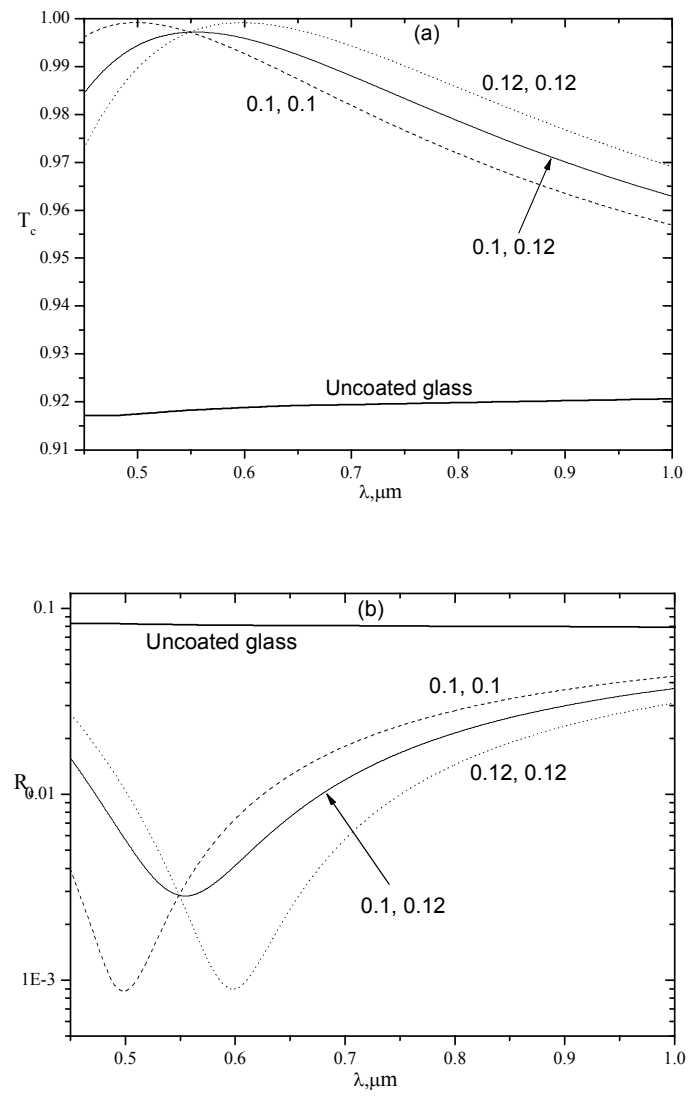

Fig. 9. Spectra of the coherent transmittance $T_{c}(a)$ and reflectance $R_{c}(b)$ of the uncoated plane-parallel glass plate (thick line) and system of highly-ordered monolayers with the hexagonal packing of $\mathrm{Al}_{2} \mathrm{O}_{3}$ particles (thin solid lines, $\left.\sigma(u)=\sigma_{0} u, \sigma_{0}=0.01\right)$ on top and bottom surfaces of the glass plate. The thickness of the glass plate is $2 \mathrm{~mm}$, filling coefficient of monolayers $\eta=0.5$. Ciphers near the lines before and after comma indicate diameters of $\mathrm{Al}_{2} \mathrm{O}_{3}$ particles (in micrometers) of top and bottom monolayer, respectively.
Consider the multilayer system (Fig. 7) in which the top and bottom surfaces of the glass plate are coated with monolayers containing spherical particles of different diameters (which are monodisperse in each monolayer). Fig. 9 shows the transmittance and reflectance spectra of a multilayer system when the surfaces of the glass plate are coated with monolayers with a hexagonal lattice of particles. As it follows from the results of calculations presented in the figure, if the top and bottom interfaces of the plate have different coatings, the spectral dependences of coherent transmittance and reflectance are more homogeneous as compared with the case of identical coatings. For the considered system, the coherent reflection coefficient in the visible spectral range is less than 0.02 with the minimum value less than 0.003 .

\section{Conclusion}

The spectra of the coherent transmission and reflection coefficients of monolayers of $\mathrm{Al}_{2} \mathrm{O}_{3}$ particles have been calculated in the wavelength range from 0.3 up to $1.0 \mu \mathrm{m}$.

Three types of coherent transmittance minima inherent for regular disperse system have been observed and described.

Coherent transmission and reflection coefficients of the glass plate coated with spherical $\mathrm{Al}_{2} \mathrm{O}_{3}$ particles have been calculated. As it follows from the results, creation of antireflection coatings do not need a high ordering of particles. Inversely, to create selective reflectors and transmission filters the arrangement of particles in monolayer has a crucial role. It is shown that the reflection coefficient can be reduced significantly by using the two-sided coating of glass plate with monolayers of alumina particles.

The obtained results can be used in the design and manufacture of the antireflection coatings, diffuse light scattering structures, transmission and reflection filters (for example, in display and solar cells applications), the lattice quality control in photonic crystals, etc.

\section{Acknowledgement}

This work was supported in part by the state scientific and technical program of Belarus "Electronics and photonics".

\section{References}

1. M. Lax, Multiple scattering of waves II. The effective field for dense systems // Phys. Rev. 85, p. 621-629 (1952).

2. J.M. Ziman, Models of Disorder. Cambridge, Cambridge University, 1979.

3. A.A. Miskevich, V.A. Loiko, Coherent transmission and reflection of a two-dimensional planar photonic crystal // J. Experiment. Theor. Phys. 113(1), p. 1-13 (2011). 
4. A.F. Skryshevskiy, Structural Analysis of Liquids and Amorphous Solids. Moscow, Vysshaya Shkola, 1980 (in Russian).

5. V.A. Loiko, A.A. Miskevich, Light propagation through a monolayer of discrete scatterers: analysis of coherent transmission and reflection coefficients // Appl. Opt. 44(18), p. 3759-3768 (2005).

6. K.M. Hong, Multiple scattering of electromagnetic waves by a crowded monolayer of spheres: Application to migration imaging films // J. Opt. Soc. Am. 70, p. 821-826 (1980).

7. C.C. Katsidis, D.I. Siapkas, General transfer-matrix method for optical multilayer systems with coherent, partially coherent, and incoherent interference // Appl. Opt, 41, p. 3978-3987 (2002).

8. E. Centurioni, Generalized matrix method for calculation of internal light energy flux in mixed coherent and incoherent multilayers // Appl. Opt. 44(35), p. $7532-7539$ (2005),

9. E.D. Palik, editor, Handbook of Optical Constants of Solids. San Diego, Academic, 1985.
10. L.S. Ornstein, F. Zernike, Accidental deviations of density and opalescence at the critical point of a single substance // Proc. Acad. Sci. 17, p. 793-806 (1914).

11. J.K. Percus, G.J. Yevick, Analysis of classical statistical mechanics by means of collective coordinates // Phys. Rev. 110(1), p. 1-13 (1958).

12. A.P. Ivanov, V.A. Loiko, V.P. Dick, Propagation of Light in Close-packed Disperse Media. Minsk, Nauka i Tekhnika, 1988 (in Russian).

13. R.W. Wood, Anomalus diffracting gratings // Phys. Rev. 48, p. 928-937 (1935).

14. N.I. Mukhurov, Aluminium-oxide Micronanostructures for Microelectromechanical systems. Minsk, Bestprint, 2004 (in Russian).

15. A.A. Lutich, M.B. Danailov, S. Volchek, V.A. Yakovtseva, V.A. Sokol, S.V. Gaponenko, Birefringence of nanoporous alumina: dependence on structure parameters // Appl. Phys. B, 84, p. 327331 (2006).

16. M. Born, E. Wolf, Principles of Optics. Cambridge University Press, 2002.

17. Corning site http://www.corning.com 